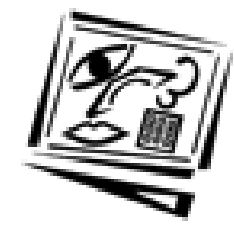

\author{
Australian Journal of \\ Educational Technology \\ 1998, 14(1), 49-59.
}

\title{
WWW support of student learning: A case study
}

\author{
Tony Greening \\ The University of Ballarat
}

\begin{abstract}
The World Wide Web (WWW) is achieving a place of prominence in educational practice. However, the benefits of using the Web to support learning are not always apparent. The most prominent public feature of the Internet is the multitude of possibilities that it presents for information retrieval. This is widely believed to offer educational advantage, although the means by which that advantage are realised are typically not well specified. The paper discusses the role of information retrieval opportunities presented by the Internet, and suggests that it requires a new model of information access best supported by a reconsideration of educational philosophy. The constructivist position is favoured. The paper also discusses tissues in using the Internet to deliver courses, arguing that the delivery model does not take full advantage of the new possibilities offered by the technology. It then presents a case study of the use of the Web in a first year computer science course, offered in a Problem Based Learning (PBL) mode. The focus is on the appropriate use of the technology as a pedagogical tool in higher education. In the case of a curriculum clearly founded on constructivist principles an important factor in the appropriateness of the supporting technology was that it did not encourage staff and students to adopt more familiar, instructivist patterns of behaviour. In this sense, the role of the Internet within the curriculum needed to be different to those roles that currently tend to typify it.
\end{abstract}

\section{Introduction}

Increasingly the role of computer technology is a networked one. Networks of networks, or "internets", participate in that global mesh of connectivity referred to as the (capital "I") Internet. At the physical level the Internet is a collection of communication media which enable paths of connectivity to be formed between remote computers. At higher levels of abstraction the Internet is concerned with software, some of which is essential to the control of Internet traffic, and others which use those protocols to deliver applications that operate in this distributed environment. The applications, while certainly providing global access 
to information and data, have not always upheld the principles of userfriendliness or integration that mark a mature software technology. The WWW helped bridge that gap, and presented a marketable Internet that was immediately accessible to the public. It both encompassed preexisting modes of accessibility and delivered new capabilities, all within an intuitive interface. The possibilities excited an already interested public and helped transform Internet observers into active participants. This paper, in discussing the Internet and the WWW, refers to them in the highest levels of abstraction. It is in the appropriate use of these enabling technologies that is of interest. How might the possibilities offered by the technology be used to support meaningful learning?

There appears to be a perception propagated by the popular media that the potential gush of traffic that is generated from Internet connectivity is going to improve the way people learn. The mechanism for that improvement is often left unspecified. Let us accept that the Internet is changing the way we approach many day-to-day tasks. It is not clear, however, to what extent we can ascribe to that change valuable shifts in the manner in which learning takes place.

It has been argued that the mechanism for interaction with this powerful tool is somewhat primitive; Crossman (1995) refers to it as COIK - Clear Only If Known. Certainly, the WWW interface has gone a long way towards addressing this issue, and continues to do so. However, improved access to information comes at a cost of increased difficulty in filtering the volume of information located. Clifford Stoll (1995, p.197) offers the following criticism of the WWW:

These hypertext documents lack unity: one page may be dense with information, another may be a vacuum. Its advocates speak of nonlinear text - you don't read a straight line of text, but can jump around as you wish. As a result, the author's logic, structure, and reasoning disappear. You get random facts.

The World Wide Web seems well-tuned for automated key-word searches. Your computer combs through files, picking out words in titles or text. It feels like you're riding atop the world's finest index. You're not.

Key-word-in-context lists are weak substitutes for a genuine index. Such searches profane the whole idea of research. In their facile expediency, they deliver information too effortlessly to be trustworthy. I know neither the depth nor breadth of the search: what databases did I miss?

Random? Weak? Facile? These are not the adjectives which are commonly used to describe the Internet, and which typically glorify it as 
a source of valuable information. However, Stoll is not a lone voice in his criticism. McKenzie (1995) warns that access to information is not the same as access to information of value, and the Internet does not always make one's position regarding the latter very clear. Searches may be approached with the wrong strategy, contain unknown biases, or provide no feedback as to the coverage achieved. Butler (1995) states that students find large, ill-structured masses of information such as that represented by the WWW very confusing, and require some degree of orientation within the knowledge domain before they become capable of extracting the information of value from such structures.

The position taken in this paper is that the Internet is, as commonly claimed, a remarkable piece of educational technology made all the more palatable by the WWW. It is a wonderful cognitive tool. However, as with any tool, it requires a level of expertise to gain full benefit from its usage. Unfortunately, the ease of use and general seductiveness of the interface may suggest that this is not the case. Certainly, the arguments presented by people such as Stoll, McKenzie and Butler cannot be dismissed. Clearly, under such circumstances as they describe, use of the Internet may result in the development of misconceptions in the knowledge domain being investigated. This is likely to be more so if the Internet is regarded as an unbiased, authoritative source. As a result, the Internet may not be the technology of choice for those who seek to maintain an objectivist epistemology or a preference for teacher-centred pedagogy; such approaches tend to value simplification (in order to reduce confusion) and the careful structuring of content to mirror external reality. The Internet is much more likely to be valuably employed in learning environments that foster more student-centred exploration of a spectrum of issues.

The description of the Internet (and other hypertext technologies) as a "cognitive tool" applies not so much to the operational aspects of the WWW, but rather towards gaining a sense of orientation within the knowledge domain in which the user may interpret the information they receive. Mayes (1994) suggests that browsing is of uncertain value until a threshold acquaintance with the domain is attained. However, beyond this threshold the benefits of browsing become apparent as students move to strategies of independent and active learning.

The discussion above has focussed on what appears to be the default public perception of the benefits of Internet connectivity, namely information retrieval. Policy makers talk principally of access to vast 
distributed databases being used to support educational projects, of school children emailing content specialists to seek their expertise, and of up-to-the-minute currency of information being extracted. This criticism of the prevailing information retrieval perspective levelled here is that it does not fully recognise that opening of increased possibilities for information retrieval has been accompanied by a change in the nature of the value of that information. It requires additional skills in order to critically process the findings obtained from information searches. However, it also suggests the need for a change in educational philosophy, away from approaches driven by authoritative sources and towards a focus on the process of navigating and critically evaluating a database of immense proportions with rapidly shifting data of uncertain value. Constructivism is a philosophy - and one with increasing presence - which embraces such a shift in emphasis.

The remainder of the paper explores other aspects of educational uses of Internet connectivity. The next section discusses the use of the Internet as an instructional medium. While there are many reasons for the adoption of WWW technology as a course delivery system (such as for distance education, or for reasons of cost-effectiveness), the emphasis is on pedagogical issues. Again, the default position is an objectivist one, perhaps even implied by the term "instructional". The final section presents a short account of the use of the WWW within a computer science course that takes a constructivist approach to education. It demonstrates a use of the Internet which is neither founded on information retrieval or course delivery.

\section{Why offer courses via the WWW?}

Much of the above discussion hinges on the anarchic nature of the Internet, to what Ibrahim and Franklin (1995) refer to as "an open corpus of material that was not necessarily meant for educational use", although it was not suggested that it was without educational use. Here, we turn briefly to consider the "closed corpus" case, in which educational courses and material are provided on the WWW.

The WWW is now a part of a set of cognitive tools that have been embraced by a distributed global community with a passion. It is here to stay. When Strommen and Lincoln (1992) comment on the technological changes that are being widely adopted by students but noticeably absent within the educational system, they are offering a comment on the 
dangers of allowing too great a rift to develop between the cognitive tools of choice and those used in education. With less powerful computer-based tools, the effect of removing them from places of instruction have been noted as severe (Duncan, 1987). More recently, Means and Olson (1995) have observed the legitimating effect that the simple inclusion of current technologies has on the performance of learning tasks. So it is not with a sense of flippancy that a first answer to the question as to why offer courses on the WWW is simply "because it is there". However, the mesmerising effect of the technology is noted, and the opportunities for being misled recognised as being many. Atkins (1993) finds that many students are seduced by the peripheral effects of multimedia and welcome the use of the technology on that basis, rather than benefiting from the learning advantages that it has been claimed to offer.

$\mathrm{Ng}$ (1994) was able to demonstrate that conversion of lecture slides to reside on the WWW, with suitable links embedded, were well received by students in a computing course, with no disorientation effects reported. The fact that the lecture notes were for a computing course may indicate that the results may not have wider application. It is suspected, however, that so far as the interface technology is concerned the bias may only be marginal; the ubiquitousness of computing technology is such that it is essentially de-mystified, and only low levels of operational expertise are required in order to manipulate the hypertext-like interface. $\mathrm{Ng}$ 's project demonstrates the two facets which Ibrahim and Franklin associate with WWW usage within a "closed corpus" of knowledge, and that is the use of hypermedia and the benefits to distance education modes of learning. These represent powerful reasons for the development of WWW courseware.

Much of the success of Ng's study may be attributed to the care with which the hyperlinks were established. Personal experience suggests that this is not always the case. More generally, instructional designers either do not always appear to take advantage of the hypermedia technology, or do so without pedagogical foundation. Phillips (1992) finds a number of common faults with the design approaches used in software development of hypermedia ranging from inflexible instructivist design in which the goal appears to be to walk in the steps of the author, to the use of inappropriate metaphors in the development. Both seem to lead to flat results in which the learning opportunities are linear and uninspiring, with an apparent attempt at compensation by use of multimedia. The use of multimedia to evoke vivid images to assist 
in association of concepts is a useful device, but it comes with a warning that is often disregarded; that which constitutes "vividness" means different things to different people (Mayes, 1994). While multimedia is a potent attribute for enhancing good pedagogical design in educational technology, it cannot be regarded as a safe (or enduring) substitute for it. Spiro, Feltovich, Jacobson and Coulson (1991) note that objectivist approaches to dealing with ill-structuredness tend to fail, resorting to over-simplification as a means of dealing with the complexity. As a result, the student misses out on the opportunity to engage in potentially rich learning environments. They note that the flexibility that current educational technology offers is such that it can offer support to nonlinear models of learning, such as that exhibited by hypertext. It is a sadly wasted opportunity, then, when linear models of instruction are transplanted onto such technology. Alexander's (1995) postmortem on failed uses of educational technology supports this argument, stating that many instructional designers focus on the technological features, and simply present old learning experiences in this new clothing.

There is a hint of a suggestion in this to practice a kind of economy of exposure of the special attributes associated with a technology. Winn (1993) in the context of discussing educational applications of virtual reality makes the point more explicit, proscribing against the use of VR to explore concepts that are capable of exploration without the assistance of VR technology. This may be a good rule of thumb to adopt in the development of educational material, encouraging the examination of features offered by technology in context of pedagogical guidelines which may productively exploit those features and, in turn, be supported by them.

This takes us full-circle, to return to the question "Why offer WWW courses?", except that now it is offered as a guide to practice rather than a question to which a static answer can be provided. That is, developers should consider asking this question in terms of the educational and logistical goals that they are addressing, and the pedagogical framework within which they are operating.

\section{A report on the use of the WWW in a PBL course}

Phillips (1992), in criticising multimedia courseware as author-centred, argued that in fact authoring offers its own benefits (requiring consolidation of previously learnt material, for example) which is commonly denied to students in the instructivist model. Confrey (1990) 
also argued that conventional (objectivist) approaches to education, withhold the process of simplification itself from students, regarding it as residing in the realm of the instructor. Thus, not only do they present overly simplified accounts of ill-structured knowledge domains, but they do so at the cost of depriving the students of benefiting from the exercise of simplification itself.

Cox and Clubb (1995, p.5) in describing the development of the implementation of quizzes on the WWW notes that one of the specifications for the development was that "there should be owners of quizzes and questions, but anyone can be a quiz and question owner." This brings us to a position where we can appreciate a use of the WWW in which students can be exposed to a number of benefits associated with authoring their own documents, and which we have implemented in a trial program of problem-based learning in a first- year computing science course (Greening, Kay, Kingston \& Crawford, 1996, 1997). PBL is an approach to learning in which tasks are used to motivate the learning rather than assess it. The learning environment in a PBL classroom emphasises group-based, student-centred learning that incorporates generic skills (time management, leadership, etc.).

In developing the PBL approach we were implementing a number of concepts which were taking us to a constructivist approach to first-year computer science. While the concepts were not new, the implementation in first-year computer science was regarded as an innovation. This involved breaking from the linear, teacher-based, curriculumconstrained model of traditional instruction, and establishing a studentcentred model in its place. One of the recurring themes in constructivist pedagogy is collaborative work, and we allowed students to select from a range of problems that they would tackle as a team over the course of a full semester. However, we were concerned at the possibility of group instability in the first few weeks of semester as a result of students leaving computer science. To address this concern we implemented a four-week session of two mini-problems, both of which involved using the WWW. The mini-problems were team-oriented in the sense that they relied upon peer collaboration, but the groups were rotating rather than fixed. Thus, students may work with different personnel at each meeting. This effectively solved the potential group instability problem, while providing a mechanism by which we could facilitate the functioning of group processes. One of the risks in adopting a PBL approach is assuming that students could easily work in groups and 
exercise independent learning. The WWW-focussed mini-problems allowed a period in which these skills could be developed.

The decision to use WWW support in this phase was a fairly simple one. An early reason was the attractiveness of the technology; this relates to the "because-its-there" factor referred to above. Students were webaware, and some of them had regular usage of the WWW in the sense of using web browsers. In retrospect, despite not having thought of it in these terms, it may have been a legitimising activity. We had a chance to offer an exciting technology to students for whom that exposure was entirely appropriate.

However, we did not have the time resources available to develop anything that may have resembled WWW course material (Pennell and Deane, 1995), and in any case would have able to convince ourselves that this may have trapped us into objectivist-like structures from which we were seeking to change.

The solution was to offer control of the WWW to the students. They were asked to construct a home page as one mini-problem, and to create a minimalist UNIX (operating system) manual as another.

Construction of a home page was a task that was student-centred and yet required the assistance of peers in order to negotiate the use of HTML. We were conscious of not satisfying the students' expectations of us as fonts of all wisdom, and this very quickly resulted in the establishment of independence, teamwork, and (importantly) problem ownership. Students were excited about the ease with which they could create their own cyberspace identity, without being fettered by a set of teacher-imposed instructions. Students' home pages were externally visible (i.e., not confined to an intranet) and this may have contributed to the appeal of the task, as well as its authenticity. A number of the students quickly became local experts on HTML, and would be found dabbling in related aspects of the technology; certainly, a curiosity for Javascript was aroused in some, and small examples started to be incorporated. The task also introduced something of the Internet "culture" of re-use, which in other environments may have been called "cheating" or "plagiarism". While we expected acknowledgment of reused work, we found that this environment provided a sense of freedom and an accompanying sense of responsibility. Cooperative work was generally acknowledged, and student ownership of the problem was evident. 
WWW support of our PBL program could then be recognised as something that was in the hands of the students. Having the skills which they gained from this problem allowed us to use the WWW in the sense that students could be asked in their main problem to, say, provide examples of some code that they have written, or to prepare a progress report. They could simply write these in HTML and link them to their homepages, where we could access them. In truth, this was not a service that was used a great deal for this purpose; more often, students would use the same approach for their own purposes. For example, one of the later (large) problems was to break classical cryptographic codes. Some groups placed their own encrypted code on a link to their home page, and challenged other students to decrypt it. This was, for us, a very satisfying use of the WWW technology to support a PBL program.

The second mini-problem asked them to create a minimalist manual for first-time UNIX users (a category which applied to almost all of them), which they would link to their home pages. They were fully responsible for their use of HTML to create hypertext links, in negotiation with their peers. This was an interesting exercise in learning UNIX, in which they were at the helm and responsible for the simplification process which normally occurs at the hand of the instructor.

There was some observation of students using the WWW for collaborative work, as suggested by Alexander (1995), although mostly they were able to use more appropriate technology supplied by the UNIX environment.

Feedback from students supported the observation that this use of the WWW offered valuable support for our PBL trial, in a manner which did not threaten to drag us (staff and students) back into more familiar instructivist roles. The technology was exploited for its properties, and it was able to assist first-year students adapt to learning roles where they were empowered to take ownership of the learning tasks, to collaborate constructively with their peers, and to become actively engaged with the material. Pedagogy is shifting towards this emphasis on studentcentred, active learning, and technology such as the WWW is expected to play a role; that role is currently ill-defined, but needs to satisfy a demand for appropriateness. Riddle, Nott and Pearce (1995) report mixed reactions by academic staff towards the role that WWW technology has to offer the future of student learning. It is in the appropriate use of such technology that fears of the viability of its place in student-centred learning may be allayed. 


\section{Acknowledgments}

The use of the WWW in the trial PBL course was strongly advocated by Judy Kay, and it is safe to say that without her persistence it would not have been granted the prominence that it received. Allan Ellis provided helpful review of this paper, and his feedback is greatly appreciated. Anonymous reviewers for AJET made some important suggestions for improvements in the final version of the paper.

\section{References}

Alexander, S. (1995). Teaching and learning on the World Wide Web. [Abstract of presentation at AusWeb'95], [Online]. Available: http: / / www.scu.edu.au:80/ ausweb95 / papers / education2 / alexander

Atkins, M.J. (1993). Theories of learning and multimedia: an overview. Research Papers in Education, 8(2), 251-271.

Butler, B.S. (1995). Using the World Wide Web to support classroom-based education: opportunities and challenges for IS educators. [Online] Available: http: / / www.gsia.cmu.edu/bb26/ papers/aiswww

Confrey, J. (1990). What constructivism implies for teaching. In Constructivist Views on the Teaching and Learning of Mathematics, National Council of Teachers of Mathematics (pp.107-122), Reston (Virg).

Cox, K. \& Clubb, O. (1995). Formative quizzes and the World-Wide Web. [Paper presented at ASCILITE'95], [Online]. Available: http: / / ascilite95.unimelb.edu.au:80/SMTU / ASCILITE95 / papers / Cox.pdf

Crossman, D.M. (1995). The Internet in higher education. In G.J. Anglin (Ed.), Instructional Technology: Past, Present and Future, (2ed). Libraries Unlimited: Englewood.

Duncan, K.W. (1987). Using computers in education intelligently: what happens when computers are removed from the classroom? In J. Barrett \& J. Hedberg (Eds), Using Computers Intelligently in Tertiary Education. ASCILITE: Kensington (NSW).

Greening, T., Kay, J., Kingston, J.H., \& Crawford, K. (1996). Problem-Based Learning of first-year computer science. Proceedings of the First Australasian Computer Science Education Conference (ACSE'96). pp. 13-18. ACM Press: Sydney.

Greening, T., Kay, J., Kingston, J.H., \& Crawford, K. (1997). Results of a PBL trial in first-year computer science. Proceedings of the Second Australasian Computer Science Education Conference (ACSE'97). pp. 201-206. ACM Press: Melbourne.

Ibrahim, B., \& Franklin, S.D. (1995). Advanced educational uses of the World-Wide Web. [Online]. Available:

ftp: / / cui.unige.ch/VISUAL_PROG/articles/WWW95.ps.gz 
Mayes, T. (1994). Hypermedia and cognitive tools. [Online]. Available: http: / / www.icbl.hw.ac.uk/ctl/mayes / paper9.html

McKenzie, T. (1995). Grazing the net: raising a generation of free range students. [Institute for Learning Technologies], [Online]. Available: http: / / www.ilt.columbia.edu/k12/livetext/ docs/graze.html

Means, B. \& Olson, K. (1995). Technology's role within constructivist classrooms. Paper presented at the Annual Meeting of the American Educational Research Association (San Francisco, CA, April 18-22, 1995).

$\mathrm{Ng}$, S.E. (1994). Distance learning using a multimedia network system. [BSc honours thesis, School of Computing, Curtin University], [Online]. Available: http:/ / www.cs.curtin.edu.au/ ngsej/thesis/thesis.ps

Pennel, R., \& Deane, E.M. (1995). Web browser support for Problem-Based Learning. [Paper presented at ASCILITE'95], [Online]. Available: http:/ / ascilite95.unimelb.edu.au:80/SMTU / ASCILITE95/ papers/ pennell.pdf

Phillips, R.L. (1992). Opportunities for multimedia in education. In S. Cunningham \& R.J. Hubbold (Eds), Interactive Learning through Visualization: The Impact of Computer Graphics in Education. (pp. 25-35). Springer-Verlag: Berlin.

Riddle, M.D., Nott, M.W., \& Pearce, J.M. (1995). The WWW - opportunities for an integrated approach to teaching and research in science. [Paper presented to AusWeb'95], [Online]. Available:

http: / / www.scu.edu.au:80/ausweb95/ papers/education1/ riddle/

Spiro, R.J., Feltovich, P.J., Jacobson, M.J., \& Coulson, R.L. (May, 1991). Cognitive flexibility, constuctivism, and hypertext: random access instruction for advanced knowledge acquisition in ill-structured domains. Educational Technology (pp. 24-33).

Stoll, C. (1995). Silicon Snake Oil. Doubleday: New York.

Strommen, E.F., \& Lincoln, B. (1992). Constructivism, technology, and the future of classroom learning. [Institute for Learning Technologies], [Online]. Available: http: / / www.ilt.columbia.edu/k12/livetext/docs/ construct.html

Winn, W.A. (1993). A conceptual basis for educational applications of virtual reality. [Technical Report TR-93-9, Human Interface Technology Laboratory, University of Washington], [Online]. Available:

http: / / www.hitl.washington.edu:80 / publications / tech-reports / tr-93-9winn.html

Tony Greening

School of Information Technology and Mathematical Sciences

The University of Ballarat

t.greening@ballarat.edu.au 